\title{
What's up with IR gluon and ghost propagators in Landau gauge? A puzzling answer from huge lattices
}

\author{
Attilio Cucchieri* and Tereza Mendes \\ Instituto de Física de São Carlos, Universidade de São Paulo, \\ Caixa Postal 369, 13560-970 São Carlos, SP, Brazil \\ E-mail: attilio@ifsc.usp.br, mendes@ifsc.usp.br
}

\begin{abstract}
Several analytic approaches predict for $S U\left(N_{c}\right)$ Yang-Mills theories in Landau gauge an enhanced ghost propagator $G\left(p^{2}\right)$ and a suppressed gluon propagator $D\left(p^{2}\right)$ at small momenta. This prediction applies to two, three and four space-time dimensions. Moreover, the gluon propagator is predicted to be null at $p=0$. Numerical studies by several groups indeed support an enhanced ghost propagator when compared to the tree-level behavior $1 / p^{2}$ and a finite infrared gluon propagator. However, the agreement between analytic and numerical studies is only at the qualitative level in three and in four dimensions. In particular, the infrared exponent of the ghost propagator seems to be smaller than the one predicted analytically and the gluon propagator seems to display a (finite) nonzero value at zero momentum. It has been argued that this discrepancy might go away once simulations are done on much larger lattice sizes than the ones used up to now. Here we present data in three and four space-time dimensions using huge lattices in the scaling region, i.e. up to $320^{3}$ at $\beta=3.0$ and up to $128^{4}$ at $\beta=2.2$, corresponding to $V \approx(85 \mathrm{fm})^{3}$ and $V \approx(27 \mathrm{fm})^{4}$. Simulations have been done on the IBM supercomputer at the University of São Paulo.
\end{abstract}

The XXV International Symposium on Lattice Field Theory

July 30-4 August 2007

Regensburg, Germany

\footnotetext{
*Speaker.
} 


\section{Introduction}

In the Gribov-Zwanziger confinement scenario for Landau gauge [1,2] the gluon propagator $D\left(p^{2}\right)$ is predicted to be infrared (IR) suppressed at small momenta. In particular, one should have $D(0)=0$, implying that reflection positivity is maximally violated. This violation of reflection positivity may be viewed as an indication of gluon confinement [3]. At the same time, the GribovZwanziger [1, 4] and Kugo-Ojima [5] confinement scenarios predict (in Landau gauge) a ghost propagator $G\left(p^{2}\right)$ enhanced in the IR limit. This represents a long-range effect and could be related to quark confinement $[1,3,4,6]$.

Several analytic studies $[7,8,9,10]$ agree with the above scenarios predicting, for small momenta, a gluon propagator $D\left(p^{2}\right) \propto p^{2\left(a_{D}-1\right)}$ and a ghost propagator $G\left(p^{2}\right) \propto 1 / p^{2\left(1+a_{G}\right)}$, with the relation $a_{D}=2 a_{G}+(4-d) / 2$. Here $d$ is the space-time dimension. Clearly, if $a_{D}>1$ one has $D(0)=0$. In the four-dimensional case, one finds $[8,9] a_{G} \approx 0.59$ and $a_{D}=2 a_{G}$. Similar power behaviors have also been obtained for the various vertex functions of $S U\left(N_{c}\right)$ Yang-Mills theories $[9,11,12]$.

These results have been confirmed at the quantitative level in the two-dimensional case, using lattices up to almost $(43 \mathrm{fm})^{2}$ [13]. In the three-dimensional case [14], one clearly sees an IRsuppressed gluon propagator. However, using lattice volumes up to about $(24 \mathrm{fm})^{3}$, it was not possible to control the extrapolation to infinite volume. In particular, the data for the rescaled gluon propagator at zero momentum $D(0)$ could be fitted [as a function of the inverse lattice side $1 / L=1 /(N a)$ ] using the Ansatz $d+b / L^{c}$ both with $d=0$ and with $d \neq 0$ [14]. Here $a$ is the lattice spacing and $N$ is the number of lattice points per direction. Finally, in four dimensions, the gluon propagator is clearly less divergent than in the tree-level case $[15,16,17,18]$. On the other hand, even using lattices with a lattice side of about $10 \mathrm{fm}$, one does not see a gluon propagator decreasing at small momenta [18]. One should stress, however, that the Landau gluon propagator clearly violates reflection positivity, in two, three and four space-time dimensions $[13,19,20]$. For the ghost propagator, the IR exponent $a_{G}$ obtained using analytic studies has been confirmed in $2 \mathrm{~d}$ [13], while in the $3 \mathrm{~d}$ [21] and in the $4 \mathrm{~d}[16,17,22,23]$ cases the exponent obtained using lattice numerical simulations is always smaller than the one predicted analytically. Let us also recall that, in Ref. [24], it was shown that gluon and ghost propagator for $S U(2)$ and $S U(3)$ YangMills theories are in very good agreement from momentum $p \approx 1 \mathrm{GeV}$ to about $p \approx 10 \mathrm{GeV}$. Similar results have been presented in [25]. These findings suggest that the IR behavior of these propagators is independent of the gauge group $S U\left(N_{c}\right)$, as predicted analytically [7, 8, 9].

Finally, by solving Dyson-Schwinger equations on a finite four-dimensional torus [12, 26, 27] one can show that the gluon propagator (at small momenta) seems to diverge for volumes up to about $(8 \mathrm{fm})^{4}$, develops a plateau for $V \approx(9 \mathrm{fm})^{4}$ and is IR suppressed for a lattice side larger than $10 \mathrm{fm}$. Also, the extrapolation of these results to infinite volume gives a null gluon propagator at zero momentum. At the same time, one obtains that, after eliminating (for each volume) the data corresponding to the first two non-zero momenta, the ghost propagator shows a power-law behavior and, in the infinite volume-limit, one obtains the IR exponent $a_{G}$ predicted by analytic studies [7, 8, 9].

From the above results, it seems necessary to extend present numerical simulations to very large lattice volumes in order to verify if the agreement obtained between lattice data and analytic 
results for the two-dimensional case [13] applies to the $3 \mathrm{~d}$ and $4 \mathrm{~d}$ cases as well. Here we present extensive simulations in three and in four dimensions, for the $S U(2)$ case, using huge lattices. In particular, we considered lattice sides $N=140,200,240$ and 320 in $3 \mathrm{~d}$ at $\beta=3.0$ and $N=48,56$, $64,80,96$ and 128 in $4 \mathrm{~d}$ at $\beta=2.2$. In $3 \mathrm{~d}$, the number of configurations was about $630,525,350$ and 45 , respectively for the four lattice sizes, both for the gluon and for the ghost propagators. In the $4 \mathrm{~d}$ case we have considered for the gluon propagator 168 configurations for $V=128^{4}$ and about 250 configurations for the other lattice sizes. For the ghost propagator we have 21 configurations for the largest volume and about 100 in the other cases. For the inversion of the Faddeev-Popov matrix we used the so-called point-source method [21,23]. Note that the lattice volumes $320^{3}$ at $\beta=3.0$ and $128^{4}$ at $\beta=2.2$ correspond, respectively, to $V \approx(85 \mathrm{fm})^{3}$ and $V \approx(27 \mathrm{fm})^{4}$. (See Refs. [14, 28] for details about how the physical lattice spacing $a$ has been set in the two cases.) Also note that all our runs are in the scaling region.

These simulations have been done in the IBM supercomputer at USP. This machine has 112 blades with 2 dual-core PowerPC 970 2.5GHz CPU's, a Myrinet network and about 4.5 Tflops peak-performance (occupying position number 363 in the TOP 500 list of November 2006).

\section{Results}

Considering the new data produced in the $3 \mathrm{~d}$ case together with old data from Refs. [14, 19], we tried an extrapolation to infinite volume for the gluon propagator $D(0)$ as a function of the inverse lattice side $1 / L$. The data for the propagator have been renormalized following Ref. [13]. Results are shown in Fig. 1. It is clear from the plot that there is no sign of a propagator going to zero as $L$ goes to infinity. Moreover, the data show a behavior of the type $D(0) \sim 1 / L$ and an infinite-volume extrapolation given by $D(0) \approx 2 \mathrm{GeV}^{-2}$. This implies $a_{D}=1$.

In the $4 \mathrm{~d}$ case (see Fig. 2), even considering very large lattice volumes and relatively large statistics, one cannot see a clear sign of a gluon propagator $D\left(p^{2}\right)$ decreasing at small momenta. Similar results have been presented at this conference by other groups [25, 29]. Clearly, also in this case the data suggest $a_{D}=1$. On the other hand, violation of reflection positivity is confirmed and the gluon propagator, considered as a function of the spatial separation $s$, becomes negative at $s \approx 1 \mathrm{fm}$, in agreement with Ref. [30].

We have also tried to estimate the IR exponent $a_{G}$ for the ghost propagator (in the $3 \mathrm{~d}$ and $4 \mathrm{~d}$ cases) using the Ansatz $G(p)=c / p^{2\left(1+a_{G}\right)}$ and considering, for each lattice volume, either the two smallest nonzero momenta or the third and fourth smallest nonzero momenta. Results are reported in Tables 1. As one can see, this IR exponent seems to go to zero as the infinite-volume limit is approached, in agreement with [16]. One should however notice that, for $p \approx 500 \mathrm{MeV}$, the exponent $a_{G}$ is about 0.3 , also in agreement with Ref. [16].

\section{Conclusions}

The above results leave us with several open questions. From the lattice point of view, one should of course investigate if Gribov-copy effects and/or finite-volume effects could explain our results. Let us recall that an improved gauge-fixing method [31] seems capable of reducing finitevolume effects for the gluon propagator by enlarging the set of allowed gauge transformations. At 


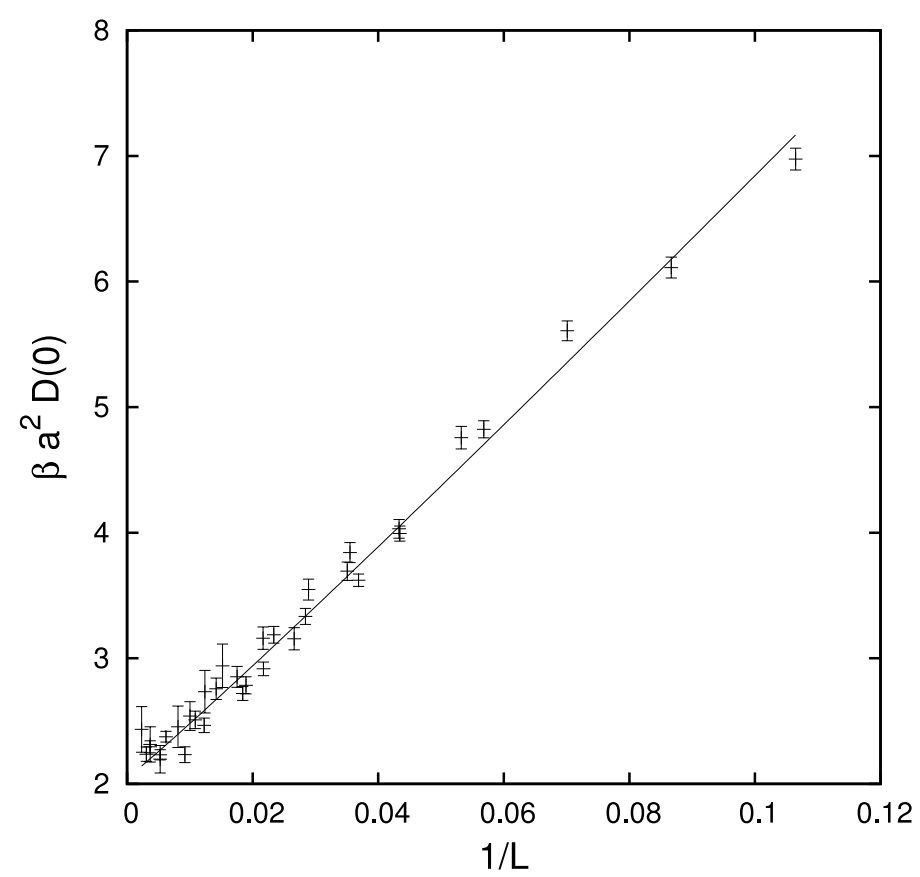

Figure 1: Renormalized gluon propagator at zero momentum $\beta a^{2} D(0)$ (in $\mathrm{GeV}^{-2}$ ) as a function of the inverse lattice side $1 / L$ (in $\mathrm{GeV}$ ) and extrapolation to infinite volume. The fit is given by $b+c / L^{e}$ with $e=1.04(5)$ and $b=2.05(5) \mathrm{GeV}^{-2}$.
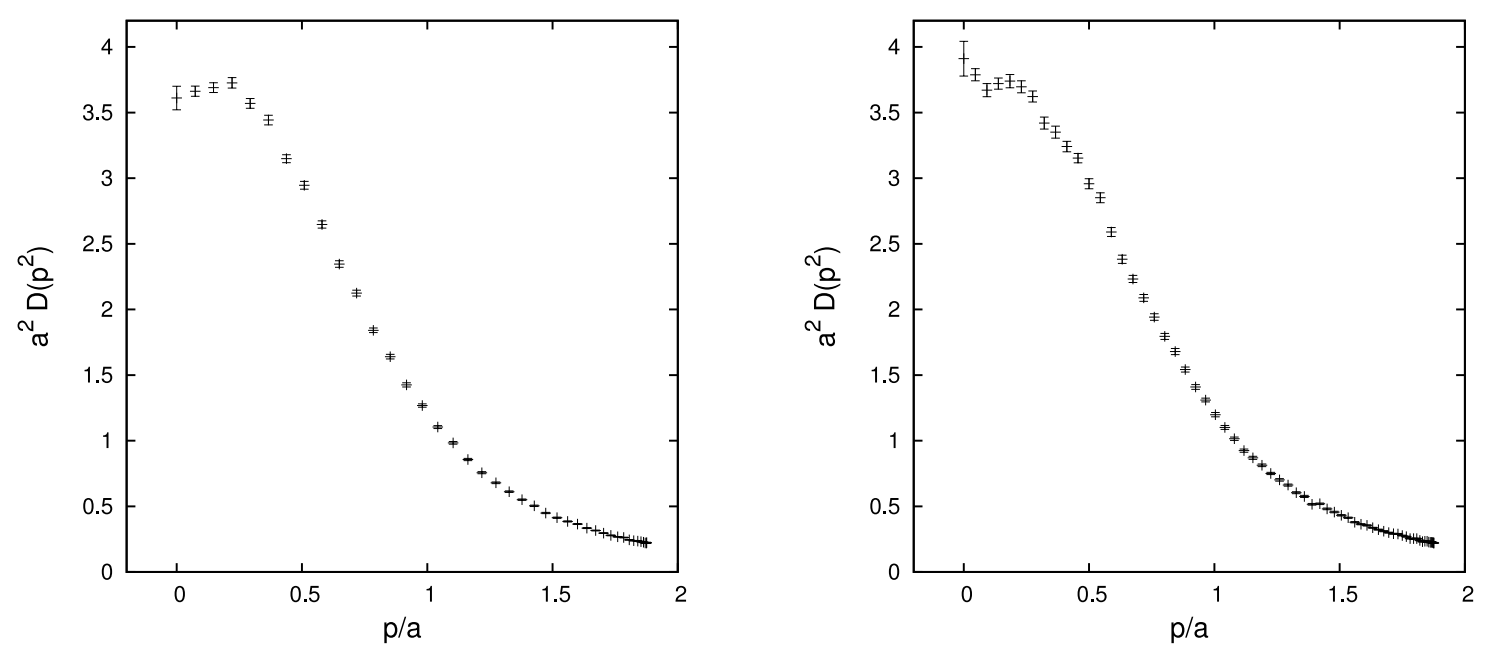

Figure 2: Unrenormalized gluon propagator $a^{2} D\left(p^{2}\right)$ (in $\mathrm{GeV}^{-2}$ ) as a function of the momentum $p / a$ (in $\mathrm{GeV}$ ) for lattice volumes $V=80^{4}$ (left) and $V=128^{4}$ (right) at $\beta=2.2$. 


\begin{tabular}{cccccccc}
$N^{3}$ & $a_{G}$ & $N^{4}$ & $a_{G}$ & $N^{3}$ & $a_{G}$ & $N^{4}$ & $a_{G}$ \\
\hline $140^{3}$ & $0.073(4)$ & $48^{4}$ & $0.093(7)$ & $140^{3}$ & $0.13(2)$ & $48^{4}$ & $0.19(4)$ \\
$200^{3}$ & $0.051(3)$ & $56^{4}$ & $0.063(6)$ & $200^{3}$ & $0.06(2)$ & $56^{4}$ & $0.18(4)$ \\
$240^{3}$ & $0.003(3)$ & $64^{4}$ & $0.049(9)$ & $240^{3}$ & $0.10(2)$ & $64^{4}$ & $0.17(4)$ \\
$320^{3}$ & $-0.021(9)$ & $80^{4}$ & $0.052(5)$ & $320^{3}$ & $0.01(5)$ & $80^{4}$ & $0.15(2)$ \\
& & $112^{4}$ & $0.038(6)$ & & & $112^{4}$ & $0.10(7)$ \\
& & $128^{4}$ & $0.016(5)$ & & & $128^{4}$ & $0.06(3)$
\end{tabular}

Table 1: Table for the ghost propagator IR exponent $a_{G}$, in the $3 \mathrm{~d}$ and $4 \mathrm{~d}$ cases, obtained using either the two smallest nonzero momenta (left) or the third and fourth smallest nonzero momenta (right).

the same time, one needs to reconcile the above results with the non-renormalizability of the ghostgluon vertex [32] and with the suppression of $D\left(p^{2}\right)$ when considering simulations in the strongcoupling regime [33], in the interpolating gauge (or $\lambda$-gauge) [34] and in Coulomb gauge [35]. From the analytic point of view, it may seem necessary to reconsider partially the conventional confinement scenarios $[1,2,3,4,5,6,7,8,9,10,11,12]$ discussed in the Introduction. One should of course recall that there are different solutions of Dyson-Schwinger equations for gluons and ghosts in Landau gauge [36, 37]. In particular, the results obtained in Ref. [36] support a finite non-zero gluon propagator and an essentially tree-level ghost propagator at small momenta. Similar results are obtained in Ref. [38]. Phenomenological tests [39] also seem to favor $D(0)>0$.

We believe that a clarification of the present status of the Kugo-Ojima/Gribov-Zwanziger scenario will probably require new ideas and new methods, both for analytic and numerical studies, and that a key point will be a better understanding of the gauge interpolating between the Landau and the Coulomb gauge [40].

\section{Acknowledgements}

The authors thank R. Alkofer, C. Fischer, H. Gies, F.J. Llanes-Estrada, A.A Natale, O. Oliveira, K. Schwenzer and S. Sorella for discussions and the Institute of Physics of the University of Graz for hospitality. We also acknowledge partial support from FAPESP, CNPq and CCInt-USP. The simulations reported here have been done on the IBM supercomputer at São Paulo University (FAPESP grant \# 04/08928-3).

\section{References}

[1] V.N. Gribov, Quantization of non-Abelian gauge theories, Nucl. Phys. B 139 (1978) 1.

[2] D. Zwanziger, Vanishing of zero momentum lattice gluon propagator and color confinement, Nucl. Phys. B 364 (1991) 127.

[3] R. Alkofer and L. von Smekal, The infrared behavior of QCD Green's functions: Confinement, dynamical symmetry breaking, and hadrons as relativistic bound states, Phys. Rept. 353 (2001) 281.

[4] D. Zwanziger, Fundamental modular region, Boltzmann factor and area law in lattice gauge theory, Nucl. Phys. B 412 (1994) 657. 
[5] T. Kugo and I. Ojima, Local Covariant Operator Formalism Of Nonabelian Gauge Theories And Quark Confinement Problem, Prog. Theor. Phys. Suppl. 66 (1979) 1 [Erratum-ibid. 71 (1984) 1121].

[6] Y.L. Dokshitzer and D.E. Kharzeev, The Gribov conception of quantum chromodynamics, Ann. Rev. Nucl. Part. Sci. 54 (2004) 487; R. Alkofer and J. Greensite, Quark Confinement: The Hard Problem of Hadron Physics, J. Phys. G 34 (2007) S3.

[7] L. von Smekal, A. Hauck and R. Alkofer, A solution to coupled Dyson-Schwinger equations for gluons and ghosts in Landau gauge, Annals Phys. 267 (1998) 1 [Erratum-ibid. 269 (1998) 182].

[8] D. Zwanziger, Non-perturbative Landau gauge and infrared critical exponents in QCD, Phys. Rev. D 65 (2002) 094039; J.M. Pawlowski, D.F. Litim, S. Nedelko and L. von Smekal, Infrared behaviour and fixed points in Landau gauge QCD, Phys. Rev. Lett. 93 (2004) 152002.

[9] C. Lerche and L. von Smekal, On the infrared exponent for gluon and ghost propagation in Landau gauge QCD, Phys. Rev. D 65 (2002) 125006.

[10] J. Braun, H. Gies and J.M. Pawlowski, Quark Confinement from Color Confinement, arXiv:0708.2413 [hep-th].

[11] C.S. Fischer and J.M. Pawlowski, Uniqueness of infrared asymptotics in Landau gauge Yang-Mills theory, Phys. Rev. D 75 (2007) 025012.

[12] C.S. Fischer, R. Alkofer, A. Maas, J.M. Pawlowski and L. von Smekal, Large volume behavior of Yang-Mills propagators, arXiv:0709.3205 [hep-lat] and these proceedings.

[13] A. Maas, Two- and three-point Green's functions in two-dimensional Landau-gauge Yang-Mills theory, Phys. Rev. D 75 (2007) 116004.

[14] A. Cucchieri, T. Mendes and A.R. Taurines, SU(2) Landau gluon propagator on a $140^{3}$ lattice, Phys. Rev. D 67 (2003) 091502.

[15] F. Gutbrod, A study of the gluon propagator in SU(2) lattice gauge theory, DESY-96-252; D.B. Leinweber, J.I. Skullerud, A.G. Williams and C. Parrinello, Gluon propagator in the infrared region, Phys. Rev. D 58 (1998) 031501; S. Furui and H. Nakajima, Infrared features of the Landau gauge QCD, Phys. Rev. D 69 (2004) 074505.

[16] A. Cucchieri, Gribov copies in the minimal Landau gauge: The influence on gluon and ghost propagators, Nucl. Phys. B 508 (1997) 353.

[17] E.M. Ilgenfritz, M. Müller-Preussker, A. Sternbeck, A. Schiller and I.L. Bogolubsky, Landau gauge gluon and ghost propagators from lattice QCD, Braz. J. Phys. 37 (2007) 193.

[18] A. Cucchieri and T. Mendes, Propagators, running coupling and condensates in lattice QCD, Braz. J. Phys. 37 (2007) 484.

[19] A. Cucchieri, T. Mendes and A.R. Taurines, Positivity violation for the lattice Landau gluon propagator, Phys. Rev. D 71 (2005) 051902.

[20] K. Langfeld, H. Reinhardt and J. Gattnar, Gluon propagators and quark confinement, Nucl. Phys. B 621 (2002) 131; A. Sternbeck, E.M. Ilgenfritz, M. Müller-Preussker, A. Schiller and I.L. Bogolubsky, Lattice study of the infrared behavior of QCD Green's functions in Landau gauge, PoS LAT2006 (2006) 076; P.J. Silva and O. Oliveira, Exploring the infrared Landau gauge propagators using large asymmetric lattices, PoS LAT2006 (2006) 075; P.O. Bowman et al., Scaling behavior and positivity violation of the gluon propagator in full QCD, arXiv: hep-lat/0703022.

[21] A. Cucchieri, A. Maas and T. Mendes, Exploratory study of three-point Green's functions in Landau-gauge Yang-Mills theory, Phys. Rev. D 74 (2006) 014503. 
[22] A. Sternbeck, E.M. Ilgenfritz, M. Müller-Preussker and A. Schiller, Going infrared in SU(3) Landau gauge gluodynamics, Phys. Rev. D 72 (2005) 014507; I.L. Bogolubsky, G. Burgio, M.

Müller-Preussker and V.K. Mitrjushkin, Landau gauge ghost and gluon propagators in SU(2) lattice gauge theory: Gribov ambiguity revisited, Phys. Rev. D 74 (2006) 034503; O. Oliveira and P.J. Silva, Exploring the infrared gluon and ghost propagators using large asymmetric lattices, Braz. J. Phys. 37 (2007) 201.

[23] Ph. Boucaud et al., Asymptotic behavior of the ghost propagator in SU3 lattice gauge theory, Phys. Rev. D 72 (2005) 114503.

[24] A. Cucchieri, T. Mendes, O. Oliveira and P.J. Silva, Just how different are SU(2) and SU (3) Landau propagators in the IR regime?, arXiv:0705.3367 [hep-lat] .

[25] A. Sternbeck, D.B. Leinweber, L. von Smekal and A.G. Williams, Comparing SU(2) to SU(3) gluondynamics on large lattices, these proceedings.

[26] C.S. Fischer, R. Alkofer and H. Reinhardt, The elusiveness of infrared critical exponents in Landau gauge Yang-Mills theories, Phys. Rev. D 65 (2002) 094008.

[27] C.S. Fischer, A. Maas, J.M. Pawlowski and L. von Smekal, Large volume behaviour of Yang-Mills propagators, Annals Phys. and arXiv: hep-ph/0701050.

[28] J.C.R. Bloch, A. Cucchieri, K. Langfeld and T. Mendes, Propagators and running coupling from SU(2) lattice gauge theory, Nucl. Phys. B 687 (2004) 76.

[29] I.L. Bogolubsky, E.M. Ilgenfritz, M. Müller-Preussker and A.Sternbeck, The Landau gauge gluon and ghost propagators in $4 d S U(3)$ gluodynamics on large lattice volumes, these proceedings.

[30] R. Alkofer, W. Detmold, C.S. Fischer and P. Maris, Analytic properties of the Landau gauge gluon and quark propagators, Phys. Rev. D 70 (2004) 014014.

[31] I.L. Bogolubsky et al., Improved Landau gauge fixing and the suppression of finite-volume effects of the lattice gluon propagator, arXiv:0707.3611 [hep-lat] .

[32] A. Cucchieri, T. Mendes and A. Mihara, Numerical study of the ghost-gluon vertex in Landau gauge, JHEP 0412 (2004) 012.

[33] A. Cucchieri, Infrared behavior of the gluon propagator in lattice Landau gauge, Phys. Lett. B 422 (1998) 233

[34] A. Cucchieri, A. Maas and T. Mendes, Infrared-suppressed gluon propagator in 4d Yang-Mills theory in a Landau-like gauge, Mod. Phys. Lett. A 22 (2007) 2429.

[35] A. Cucchieri and D. Zwanziger, Numerical study of gluon propagator and confinement scenario in minimal Coulomb gauge, Phys. Rev. D 65 (2002) 014001.

[36] A.C. Aguilar and A.A. Natale, A dynamical gluon mass solution in a coupled system of the Schwinger-Dyson equations, JHEP 0408 (2004) 057.

[37] Ph. Boucaud et al., The infrared behaviour of the pure Yang-Mills Green functions, arXiv:hep-ph/0507104.

[38] M. Frasca, Infrared Gluon and Ghost Propagators, arXiv:0709.2042 [hep-th] .

[39] A.A. Natale, Phenomenology of infrared finite gluon propagator and coupling constant, Braz. J. Phys. 37 (2007) 306.

[40] C.S. Fischer and D. Zwanziger, Infrared behaviour and running couplings in interpolating gauges in QCD, Phys. Rev. D 72 (2005) 054005. 